\title{
UNA PROSPECTIVA DE LAS TECNOLOGÍAS DE LAS ESTRUCTURAS ESPACIALES. LOS PUENTES
}

\author{
(A PROSPECTIVE OF THE TECHNOLOGIES FOR SPACIAL STRUCTURES. BRIDGES)
}

Avelino Samartín, Ingeniero de Caminos, Canales y Puertos

Universidad Politécnica de Madrid

ESPAÑA

Fecha de recepción: $27-I V-98$

\section{RESUMEN}

El objetivo de este artículo es intentar predecir las tendencias en esta clase tan importante de estructuras espaciales. Trata en primer lugar acerca de los mecanismos para el desarrollo de los puentes. A continuación se describe la evolución de los diferentes componentes de la tecnología de los puentes y su repercusión sobre los proyectos. Posteriormente se muestran algunos ejemplos representativos de proyectos actuales de puentes.

\section{Introducción}

Los puentes son estructuras espaciales con características muy particulares, tanto de función como de diseño. Ello ha generado una cantidad exhaustiva de literatura especializada.

La función principal de un puente se orienta a resolver el conflicto producido por la intersección de dos o más corrientes de tráfico. En otras ocasiones los puentes han de salvar obstáculos, bien naturales (ríos, valles) o bien ocasionados por las actividades humanas (edificaciones). Por ello parece obvio que el origen de los puentes está ligado a las necesidades de comunicación de los primeros humanos [1]. Aunque el puente corresponde a un tipo frecuente de construcción muy próxima a la vida cotidiana, sus dimensiones a menudo provocan sorpresa y admiración según se puede deducir por los numerosos puentes existentes en el mundo con el nombre de "Puente del Diablo".

El objetivo de este artículo es intentar predecir las tendencias en esta clase tan importante de estructuras espaciales. Para

\section{SUMMARY}

The objective of this work is to predict the tendencies of this important kind of spatial structures. First it deals with mechanisms for the development of bridges. Following it describes the evolution of the different components of the bridge technology and its repercussion on the projects. Later, some representative examples of modern projects of bridges are presented. ello, resulta conveniente realizar una reflexión previa sobre la historia de los puentes y su situación en el momento actual, en particular con relación a los logros ya alcanzados.

Diversos factores interrelacionados han intervenido en el desarrollo de la tecnología de los puentes, de entre los cuales se pueden mencionar: 1) el empleo de nuevos materiales, 2) nuevas tipologías estructurales y metodologías de análisis, 3) desarrollo de nuevas técnicas constructivas y 4) demandas de uso de nuevos puentes.

Más recientemente, la concienciación mundial acerca de la limitación de los recursos naturales y el impacto de las actuaciones humanas sobre el hábitat han sido factores que han afectado a la evolución actual de los puentes. A este respecto, las consideraciones de diseño actuales de tipo ambiental, las relativas a estética, cantidad de recursos demandados e instalaciones de mantenimiento, así como otros aspectos relacionados con la eficiencia estructural, rendimiento constructivo y economía global se tienen muy presentes. Actualmente, las redes de transporte en los países 
post-industriales están prácticamente completadas, por lo que la demanda de construcción de nuevos puentes ha disminuido drásticamente. No obstante, los nuevos sistemas de transporte de masas, como los ferrocarriles de alta velocidad europeos, abren algunas perspectivas, aunque limitadas. Este hecho introduce un nuevo aspecto en el diseño de puentes, que es el de la gestión del puente. Dentro de este concepto se incluye la inspección, detección y, eventualmente, la sustitución del puente; es decir, se incorpora una nueva e importante tarea a los constructores de los puentes o "pontífices".

Este artículo trata, en primer lugar, acerca de los mecanismos para el desarrollo de los puentes. A continuación se comentan los distintos componentes, así como su influencia sobre el proceso de diseño -materiales a emplear, tipo estructural y de análisis a efectuar-y, también, la construcción y la explotación. Posteriormente se mostrarán algunos ejemplos representativos de proyectos actuales de puentes. Obviamente, en el ámbito de esta publicación no es posible realizar una presentación completa. Finalmente, se indican algunas reflexiones sobre las tendencias futuras en la tecnología de los puentes y sobre cómo éstas pueden influir sobre las técnicas actuales y sobre las actividades que los diferentes grupos de trabajo e investigación realizan sobre estas estructuras.

\section{Mecanismos de desarrollo de los puentes}

La esencia de un puente es salvar un obstáculo. Esto supone un problema técnico que, para un estado actual de materiales disponibles, de tipologías estructurales, métodos de análisis y de construcción conocidos, consiste en diseñar un puente concreto de una luz prefijada, para unas condiciones de terreno, de sustentación y de demandas de tráfico, dentro de un contexto económico dado. El abanico de posibilidades a que ello conduce es pequeño.

Cuando en la etapa de proyecto se introduce una nueva solución estructural, automáticamente aparece un desarrollo en la tecnología de los puentes. Obviamente, esto sucede en

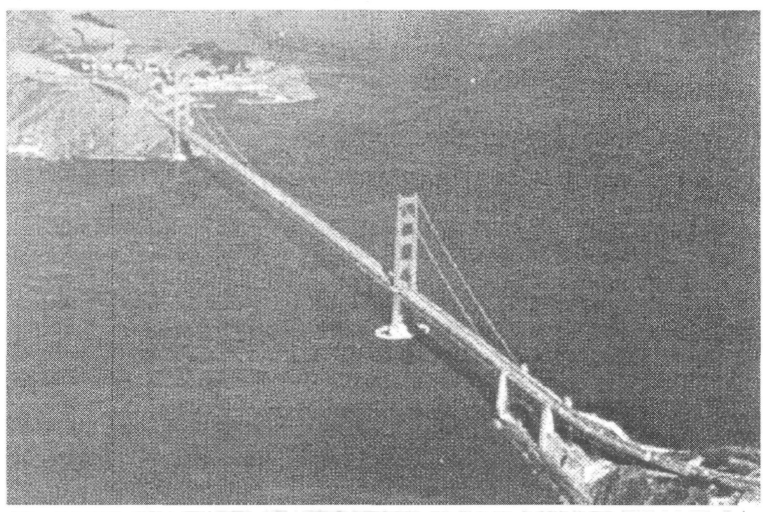

Figura 1.- El Puente Golden Gate. situaciones límite, como en casos de muy grandes luces. Actualmente, los retos que suponen los puentes de Gibraltar o de Messina, traen consigo nuevos problemas, por lo que el diseño de los mismos queda muy distante de los comentarios de Sejourné acerca de los puentes arco de piedra " el diseño de un puente es sólo una cuestión de experiencia, suficiente con observar los que se han construido en el pasado".

En el ámbito de las grandes luces, dos tipologías compiten entre sí por el primer lugar: los puentes atirantados y los puentes colgantes; no obstante, para las muy grandes luces, todavía mantiene el liderazgo la del puente Golden Gate, en San Francisco. Sin embargo, existen puentes como el Bay Bridge, que une Oakland con San Francisco, en el cual concurren ambas tipologías: el cambio de colgante a atirantado tiene lugar en la isla Yerba Buena. El vistoso puente Golden Gate (Figura 1) todavía prevalece como un símbolo de los puentes colgantes, incluso cuando su impresionante longitud de vano central, $1.280 \mathrm{~m}$, ha sido sobrepasada en las últimas décadas. Este hecho, sobradamente referenciado [2] es consecuencia de los continuos avances en la tecnología de los puentes, según se puede apreciar en la tabla 1.

El puente colgante más largo, en construcción, había sido diseñado, inicialmente, para soportar tráfico de vehículos y del ferrocarril. Tenía una longitud de vano central de 1.768 metros pero, en 1985 , se decidió orientarloúnicamente para uso como autopista, con una luz, en el vano central, de 1.990 metros, es decir, un 50 por ciento más largo que el Golden Gate. Se trata de un puente colgante de tres vanos, con doble viga riostra. La longitud total del puente es 3.910 metros y el tablero tiene 35 metros de ancho. Dicho puente ha sido proyectado por la Administración de las islas Honshu-Shikoku para una velocidad de corriente del agua de 4,5 metros por segundo, velocidad del viento de 80 metros por segundo y una magnitud del terremoto de diseño de 8,5 (escala Richter). El coste estimado en 1985 fue de 2,3 millardos de dólares. En la figura 2 se muestra este impresionante puente.

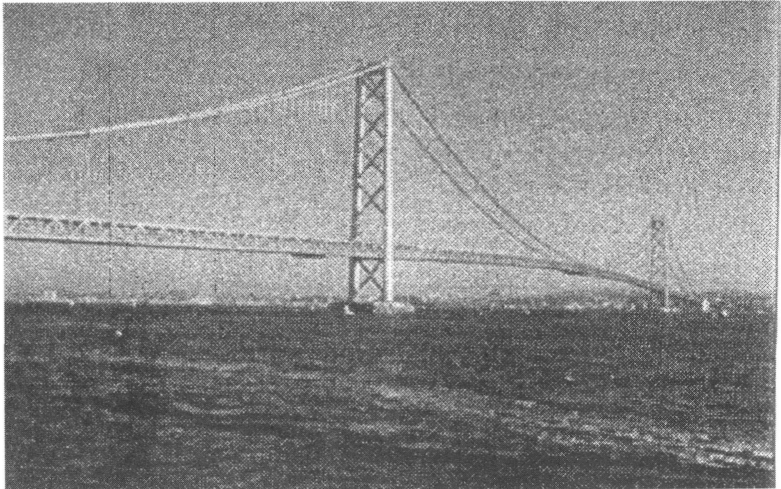

Figura 2.- Puente Akashi Kaikyo. 
TABLA 1

\begin{tabular}{|c|c|c|c|}
\hline \multicolumn{4}{|c|}{ Puentes colgantes } \\
\hline Nombre del puente & $\begin{array}{l}\text { Longitud del vano } \\
\text { central (metros) }\end{array}$ & País & $\begin{array}{c}\text { Año de } \\
\text { finalización }\end{array}$ \\
\hline $\begin{array}{l}1 \text { Akashi Kaikyo } \\
2 \text { Great Belt East } \\
3 \text { Humber } \\
4 \text { Jiangrin Br. sobre el río Yangtze } \\
5 \text { Tsing Ma } \\
6 \text { Verrazano Narrows } \\
7 \text { Golden Gate } \\
8 \text { Högsten } \\
9 \text { Estrecho de Mckinac } \\
10 \text { Minami Bisan-seto }\end{array}$ & $\begin{array}{l}1990 \\
1624 \\
1410 \\
1385 \\
1377 \\
1298 \\
1280 \\
1210 \\
1158 \\
1100\end{array}$ & $\begin{array}{l}\text { Japón } \\
\text { Dinamarca } \\
\text { Inglaterra } \\
\text { China } \\
\text { Hong Kong } \\
\text { EEUU } \\
\text { EEUU } \\
\text { Suecia } \\
\text { EEUU } \\
\text { Japón }\end{array}$ & $\begin{array}{l}1998 \text { (est) } \\
1998 \text { (est) } \\
1981 \\
1997 \text { (est) } \\
1997 \text { (est) } \\
1964 \\
1937 \\
1998 \text { (est) } \\
1957 \\
1988\end{array}$ \\
\hline
\end{tabular}

TABLA 2

Puentes atirantados

\begin{tabular}{||l|c|c|c|c|}
\hline \multicolumn{1}{|c|}{ Nombre } & $\begin{array}{c}\text { Vano central } \\
\text { (metros) }\end{array}$ & Tablero & $\begin{array}{c}\text { Año de } \\
\text { terminación }\end{array}$ \\
\hline & & & \\
1 Normandía & 856 & Mixto & Francia \\
2 Tsurumi Tsubasa & 510 & Acero & Japón & 1995 \\
3 Barrios de Luna & 440 & Hormigón & España \\
4 Annancis & 465 & Acero & Canadá \\
5 Dame Point & 396 & Hormigón & EEU \\
6 Luling & 373 & Acero & 1986 \\
\end{tabular}

La construcción de este puente ha motivado que la propuesta de T.Y. Lin de 5.000 metros en el vano central para salvar el estrecho de Gibraltar y la presentada por Freeman Fox, de 3.200 metros de vano central para cruzar el estrecho de Messina, sean cada vez más verosímiles.

La competencia entre los puentes colgantes y los atirantados se está produciendo en longitudes de vano menores. Leonhardt, entre otros, introdujo por primera vez en Alemania la tipología atirantada aplicada a la reconstrucción de las estructuras que habían quedado destruidas durante la Segunda Guerra Mundial. Su utilización y construcción se extendió a otros países, en particular por razones económicas y estéticas. Es la tipología estructural predominante en el rango de 200 a 600 metros, aunque cabe su aplicación a valores superiores. En la tabla 2 se recogen algunos de los puentes de este tipo, en la que se distingue, por su importancia, si el tablero es de hormigón o metálico. En dicha tabla se observa que el puente de mayor luz es el de Normandía; fue proyectado por Virgoleux y el equipo de la división de puentes del SETRA en París, y su tablero es una estructura mixta. El vano central, de 620 metros, consta de segmentos tipo cajón metálico ortótropo, mientras que el resto del puente está constituido por segmentos de hormigón.

En España, en las proximidades de León, se encuentra el puente de Barrios de Luna, proyectado por la Oficina Técnica Carlos Fernández Casado. Para salvar el embalse del mismo nombre, se dispuso un vano central de 440 metros y dos laterales de 66,74 metros (Figura 3). Dicha estructura constituye el puente atirantado de hormigón más largo en la actualidad. En la parte central del puente, en la que los esfuerzos axiles producidos por los tirantes son pequeños, se eliminó parte de la tabla inferior de la sección del cajón. Ambas torres tienen una altura de 90 metros respecto del nivel del tablero. Este es monolítico con el contrapeso y tiene una junta articulada deslizante en el centro del vano principal. La existencia de dicha junta hace que los momentos flectores se reduzcan notablemente en la 


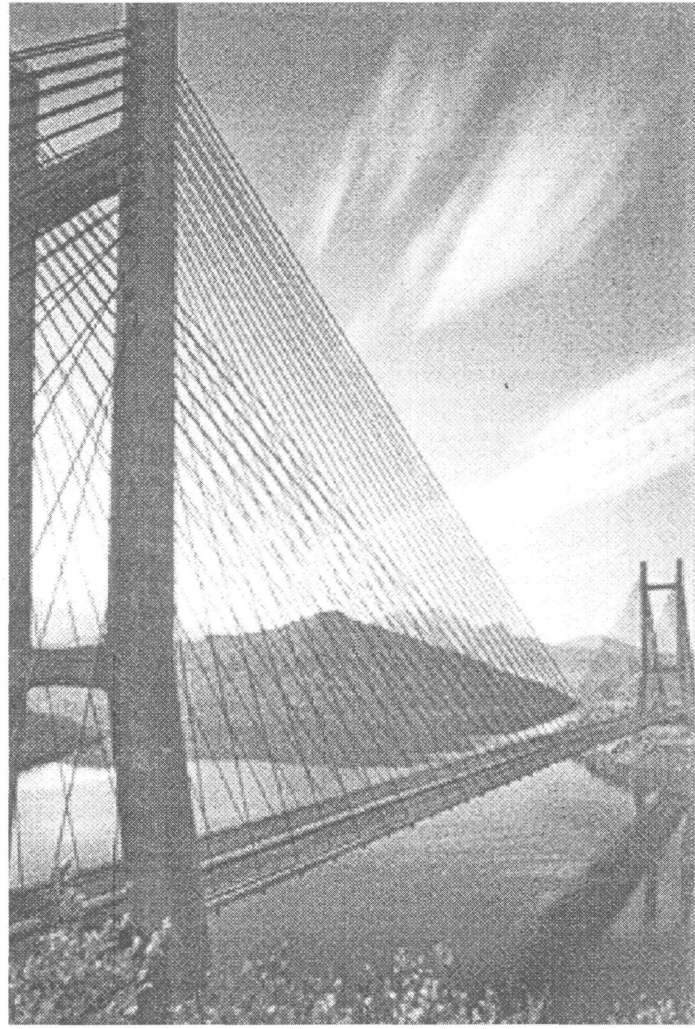

Figura 3.- Puente de Barrios de Luna.

zona central. Como contrapartida, ha de transmitir y resistir esfuerzos cortantes y de torsión, aparte de la discontinuidad que supone en la superficie de rodadura.

El puente Tsurumi Tsubasa (Figura 4), de 1.020 metros de longitud, es de planta recta y consta de tres vanos, con un vano central de 510 metros. Fue construido sobre el estrecho de Tsurumi en Yokohama (Japón). Las torres en forma de $\mathrm{Y}$ invertida tienen una altura de 163 metros respecto del nivel del tablero. La disposición de los cables es del tipo semiabanico y en un solo plano; se diseñó de esta forma con idea de construir un nuevo puente gemelo, paralelo al actual y evitar así efectos visuales no deseables. El tablero consta de una sección metálica en cajón con cinco celdas, y tiene 38 metros de ancho y cuatro metros de canto.

De lo anterior se desprende que Japón es actualmente un país muy activo en cuanto a proyecto y construcción de puentes atirantados y colgantes. Aun cuando estos se extienden por todo el país, la mayor parte (17 en total) están siendo construidos o han sido terminados recientemente para unir las dos islas principales, Hoshu y Shikoku, a través de tres puntos de cruce. El primero, con 6 carriles de autopista, consta de dos puentes colgantes: El puente de Ohnaruto (1985), de 876 metros de longitud y el ya descrito de Akashi Kaykyo. En el segundo punto de cruce existen tres puentes colgantes (con longitudes de 940,1.100 y 990 metros), dos puentes atirantados (ambos de 420 metros) y uno en celosía, para paso de 4 carriles de autopista en el tablero superior y dos líneas de ferrocarril en el tablero inferior. Todos ellos se terminaron antes de 1988. La tercera ruta consta de nueve puentes de autopista, uno de los cuales tiene una longitud de 770 metros.

A pesar que se han construido numerosos e impresionantes puentes del rango de las grandes luces, la mayoría de los nuevos avances en la tecnología de los puentes tiene lugar en las luces más pequeñas, como consecuencia de la competencia existente entre las diferentes tipologías susceptibles de ser proyectadas para un determinado emplazamiento. En dichos límites difusos, los distintos proyectos tratan de incrementar los límites inferiores o bien sus techos de aplicabilidad. Al igual que a lo largo de la Historia, los avances de las civilizaciones se han producido a lo largo de las fronteras o de las diferencias entre ellas, a través del fomento de intercambios culturales e incluso a veces a causa de disputas fructíferas, también así ha sucedido con el desarrollo en el proyecto de puentes. Un ejemplo corresponde al rango de las luces intermedias (50-70 metros), en el que compiten las soluciones de viga continua de hormigón y la metálica.

De la pugna técnica y económica entre diferentes soluciones por salvar una determinada distancia u obstáculo, se deriva un intercambio provechoso. Las formas estructurales y los métodos de construcción específicos de un determinado material en particular se transmiten a menudo a los de otro. Por ejemplo, las secciones transversales en forma de T o en cajón de los tableros de hormigón reproducen a las análogas de acero. El método de construcción de los voladizos equilibrados para puentes de hormigón sugirió un método similar para los metálicos y viceversa. Por ejemplo, en España se han construido por un procedimiento bastante similar dos puentes arco notablemente diferentes: el puente de Ricobayo (Figura 5), de estructura mixta, y el puente de La Regenta (Figura 6), de hormigón.

\section{Materiales}

Históricamente, han desempeñado un papel determinante en el diseño final del puente, al condicionar el tipo estructural y el rango de aplicabilidad. La tabla 3 muestra los principales materiales, así como los tipos diferentes de hormigón empleados en la construcción actual de puentes.

Dejando aparte la madera, la mampostería o la piedra, empleados históricamente, los que se utilizan, fundamentalmente, son el acero y el hormigón, junto con la combinación conocida como estructura mixta.

\section{(a) El hormigón}

Su limitación más importante es la relación entre resistencia y peso propio, la cual es baja en comparación con el valor análogo para el acero. Sin embargo, este último 


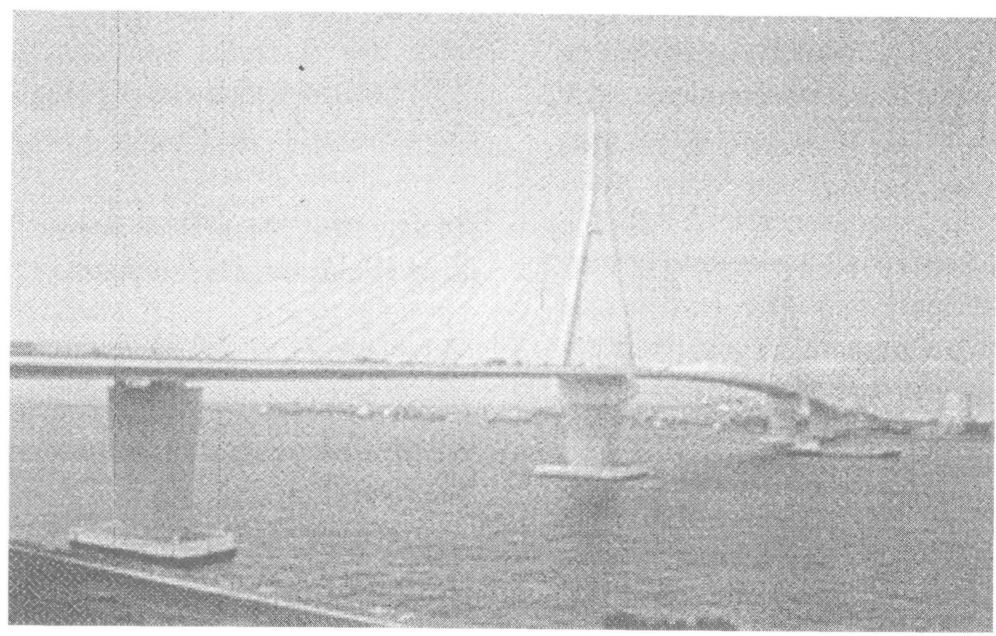

Figura 4.- Puente de Tsumuri Tsubasa.

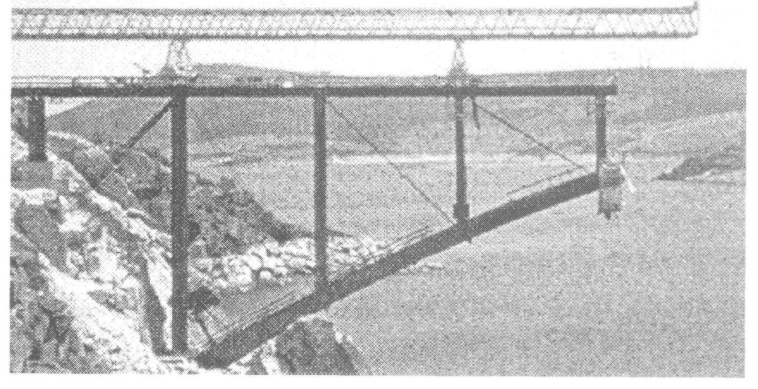

Figura 5.- Puente de Ricobayo sobre el río Esla (Ferrovial O. T.).

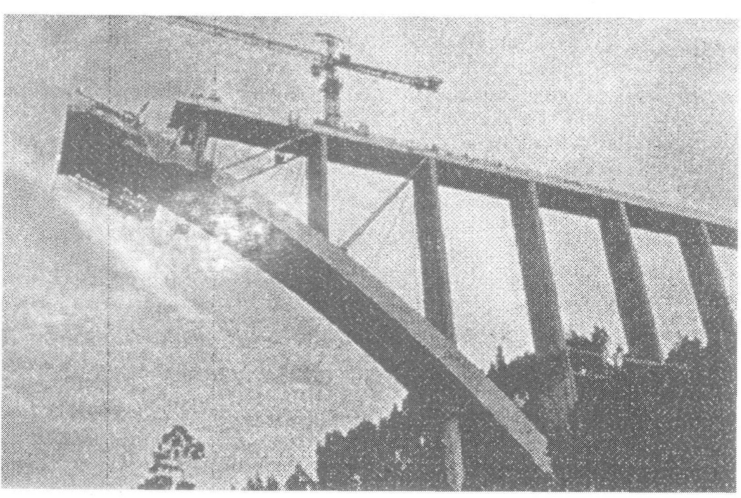

Figura 6.- Puente arco de la Regenta, en Asturias (APIA XXI).

TABLA 3

Materiales

\begin{tabular}{||c|c||}
\hline Material & Hormigón estructural \\
\hline & \\
Madera & Armado \\
Mampostería & Pretensado \\
Piedra & Postensado \\
Acero & Pretensado exterior \\
Hormigón & De alta resistencia \\
Mixto hormigón-acero & Ligero \\
\hline
\end{tabular}


adolece de mayor coste inicial y del de protección frente a la corrosión y constituyen sus mayores inconvenientes para su empleo en las luces pequeñas.

Para luces pequeñas, los tableros de hormigón parecen suficientes para resistir las cargas gravitatorias, pero han de pre o postesarse si las luces aumentan. Habitualmente estas soluciones pasan por la prefabricación, aunque otras veces se construyen in situ.

Para las luces medianas, el empleo del pretensado es muy frecuente y la tendencia creciente en su uso, se consigue mediante la utilización de hormigones de alta resistencia y hormigones ligeros, con lo que se mejora el índice resistencia/peso propio citado antes. Las posibilidades que añade el pretensado exterior en el diseño de nuevos puentes o en la reparación de los existentes, aumentan considerablemente. En concreto, el pretensado exterior confiere mayor versatilidad y posibilidad de sustitución; no obstante, todavía presenta algunos inconvenientes, tales como su menor capacidad de resistir la carga última, concentración de tensiones y dificultades para modelizar su comportamiento, todo lo cual requiere, actualmente, mayor investigación.

El futuro de los puentes de hormigón se puede deducir a partir de los hitos o récords, actualmente en vigor, para los distintos tipos de puentes [3]:

(1) Puentes atirantados: el ya citado anteriormente de Barrios de Luna (1983) que, con 440 metros, ostenta la mayor longitud.

(2) Puentes arco: el potencial de esta tipología queda bien ilustrado por los 390 metros de vano del puente San Marcos-KRK (1981) sobre el mar Adriático.

(3) Puentes en celosía: el vano central de 250 metros del puente pretensado de apoyos verticales construido para el metro de Oporto ha sido sobrepasado por otro similar de 310 metros en la misma localidad.

(4) Puentes de vigas: el récord actual lo tiene el puente Gateway, en Australia, con 260 metros de luz en su vano principal.

En conjunto, comparado con el acero, el hormigón se adapta a casi cualquier morfología y resulta estéticamente agradable. Goza de mayores ventajas en el rango de luces medianas. Para mejorar su rendimiento, en la actualidad, se recurre a: 1) empleo de hormigones de alta resistencia, ligeros $\mathrm{o}$, incluso, diferentes materiales de hormigón en la misma estructura, 2) nuevos esquemas estructurales, 3) materiales mixtos y (4) sustitución de las armaduras de acero por fibras especiales tales como kevlar o arapree.

\section{(b) Acero}

Su eficiencia estructural se muestra desde su empleo en el puente de ferrocarril de Firth of Forth (1890) en Escocia y en el de George Washington (1931), en Nueva York, que salva una distancia considerable sobre el río Hudson (1.031 metros). Para cada tipología estructural, se resumen, a continuación, los récords actuales en puentes metálicos:

(1) Puentes colgantes: el puente Humber en el Reino Unido es el más largo actualmente (1.410 metros).

(2) Puentes atirantados: el puente Annacis (Canadá) con 467 metros es ahora mismo el récord de la categoría, aunque existe un proyecto para uno similar con 780 metros cerca de El Havre (Francia).

(3) Puentes arco: el récord lo ostenta el puente de NewRiver Gorge, en West Virginia, EEUU (1980) con una longitud de 510 metros.

(4) Puentes celosía: el viaducto de Sfalassa, en Italia, (1980), para la autopista Salerno-Reggio, mantiene el récord de longitud, con 376 metros, subdivididos en tramos de 108-160-108 metros.

(5) Puentes de vigas: el puente de Niteroi (Río de Janeiro, 1975 ), con sus 300 metros de longitud.

En resumen, el acero mantendrá su liderazgo sobre los demás materiales en los puentes de gran longitud, a causa de su elevada eficiencia estructural; también resultan competitivos, económicos y adecuados para las luces grandes y medianas. Es fácil observar que cada tipología estructural de puente tiene un representante metálico de gran longitud. Sin embargo, el alto coste del acero y sus necesidades de mantenimiento restringen su empleo en el rango de las longitudes pequeñas y medianas.

\section{(c) Materiales mixtos}

El óptimo empleo conjunto en estructuras de puentes de ambos materiales- hormigón y acero- se ha convertido en una solución altamente competitiva debido a la necesidad de reducir costes y plazos de ejecución. Se pueden clasificar en dos grupos los puentes mixtos: Longitudinalmente y transversalmente mixtos. El puente de Normandía representa un ejemplo sobresaliente del primer grupo. Una parte de sus 856 metros de longitud es metálica y la restante es de hormigón. Esta composición mixta longitudinal puede ser interesante para grandes luces o bien en casos muy específicos en los que la reducción de peso propio es indispensable, como, por ejemplo, en puentes con vanos adyacentes muy desiguales. Otros casos de creciente aplicación de este grupo son los puentes mixtos atirantados. 
En el puente transversalmente mixto, ambos materiales interactúan en cada sección transversal del tablero, lo que corresponde a una solución frecuentemente adoptada en la práctica habitual [4]. Actualmente, la mayoría de este tipo de puentes pertenece a uno de estos dos tipos:

(a) Tableros mixtos con dos vigas placas: su empleo es muy extendido en Europa para puentes de autopista y de ferrocarril, como el TGV en Francia. La tendencia en estos puentes es a aumentar el espesor de las alas de las vigas, llegando incluso a más de 150 milímetros con control de calidad in situ para las uniones soldadas. Una alternativa para reducir dichos espesores tan grandes consiste en ensanchar las alas, aunque, si éstas superan los $1.000 \mathrm{~mm}$, la siguiente solución resulta más favorable.

(b) Tableros mixtos en cajón monocelular: normalmente esta solución suele ser más cara, aunque puede resultar ventajosa en grandes luces, en tableros en curva o en casos en los que se adoptan esbelteces elevadas (por consideraciones estéticas, como, por ejemplo, en puentes urbanos). En este tipo de tableros, la posibilidad de utilizar una sola pila, en lugar de varias pilas en el mismo apoyo para soportar las vigas que exige la solución (1), representa una ventaja estructural y estética. No obstante, para comparar estas dos soluciones, ha de tenerse también en cuenta que esta solución requiere mayores plazos de ejecución y que el transporte es muy caro y complicado, debido a las restricciones que presenta el tráfico rodado a los transportes de dimensiones especiales. Entre los avances recientes, sobre todo en Francia, logrados en los puentes transversalmente mixtos, se destacan:

(1) Cajones trapezoidales con alas de hormigón y almas de chapas de acero rigidizadas o celosías de acero.

(2) Cajones triangulares en los que el ala inferior se reduce a un tubo de acero (a veces relleno de hormigón) y dos almas de acero corrugado.

(3) Celosía espacial metálica con forjado superior de hormigón: un ejemplo sobresaliente es un viaducto experimental (puente Roise), proyectado por Jean Muller, en el que se emplea hormigón de alta resistencia en el forjado, además de un pretensado exterior, lo que le permite a aquél una reducción de espesor hasta los 140 milímetros.

\section{Formas estructurales}

En el proyecto de un puente se ha de considerar cualquier forma estructural. Alguna de ellas, como la columna o el muro, es específica de alguna parte del puente -pilas y estribos-. Centrándose en la superestructura del puente, en cuanto al camino que siguen las tensiones de compresión, el arco ha sido el gran invento del Arte Clásico para resistir las cargas, además de una estructura impresionante en sí misma. Como escribió E. Torroja [5]: "Si la columna es arquitectura pura, el arco es ingeniería; ..." ambos comparten la misma manera de resistir, mediante tensiones de compresión.

En la época en que la piedra era el material de construcción de puentes, el arco era la formà estructural por excelencia; esta práctica continuó hasta el advenimiento de nuevos materiales, acero y, principalmente, el hormigón. Sin embargo, la construcción de un arco no es tarea fácil, dado que se requiere disponer de todos los segmentos que lo componen, incluido el de clave, para que el arco trabaje como antifunicular del peso propio. El desarrollo de los recientes procedimientos de construcción han hecho renacer el empleo del arco. Algunos ejemplos españoles, no incluidos en los ya comentados, se muestran en las figuras 7 y 8 .

La extensión natural de la estructura arco es la membrana o, en general, la lámina, aislada o bien junto con otros elementos estructurales, con objeto de formar un sistema general de láminas plegadas. Para el caso singular del puente, cuya longitud es normalmente mucho mayor que las otras dimensiones, la lámina como elemento estructural del puente

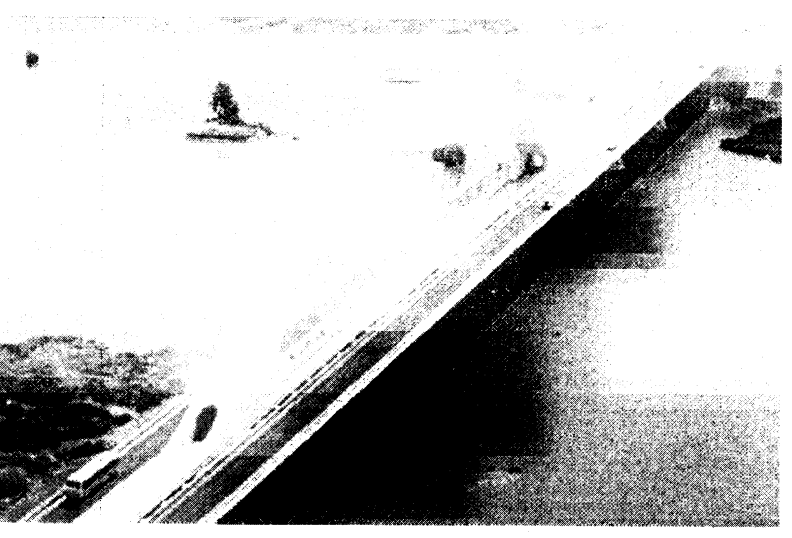

Figura 7.- Puente sobre el rio Guadiana a su paso por Mérida (Badajoz)

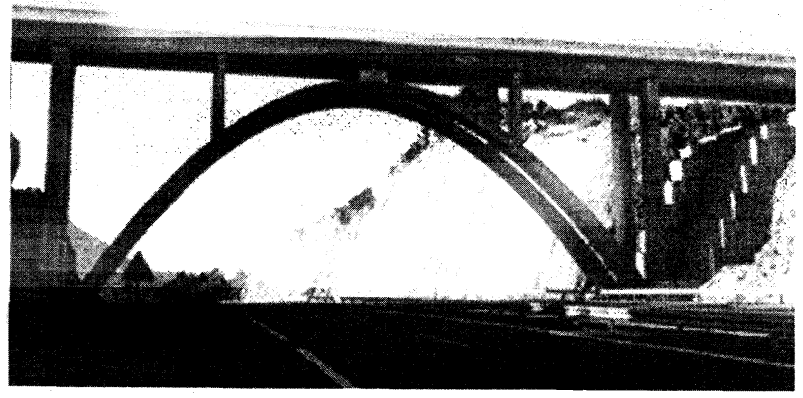

Figura 8.- Puente arco en Solares (Santander). 
no ha sido completamente explotada todavía. En cambio, otros dos elementos estructurales son muy habituales en las estructuras de los puentes: la viga, y su extensión en dos dimensiones, la placa. La flexión es una manera obvia, pero cara, de transmitir las cargas gravitatorias y del tráfico a la sustentación. Quizá sea también la forma más primitiva. Con objeto de reducir el peso propio, la viga se convierte en celosía, y la placa en emparrillado o en celosía espacial. En este proceso, los puentes de hormigón imitan a los metálicos, de tal modo que la viga y la placa pueden evolucionar hacia el puente atirantado considerado como viga continua de canto variable (Figura 9). En este sentido, cabe mencionar el viaducto La Arena (Vizcaya), multivano atirantado, y el puente de Osomort, con pretensado extra dorsal, o las nuevas tendencias hacia secciones de tablero en T invertida.

Esta reducción del peso propio se puede abordar a nivel de sección, es decir, mediante sección transversal en cajón o, en cambio, mediante una lámina plegada. Obviamente, es posible también la combinación de dichos elementos estructurales, por lo que se pueden proyectar los puentes como vigas continuas, estructuras mono o multiporticadas, estructuras de tipo Maillart, vigas ménsula, etc. Como

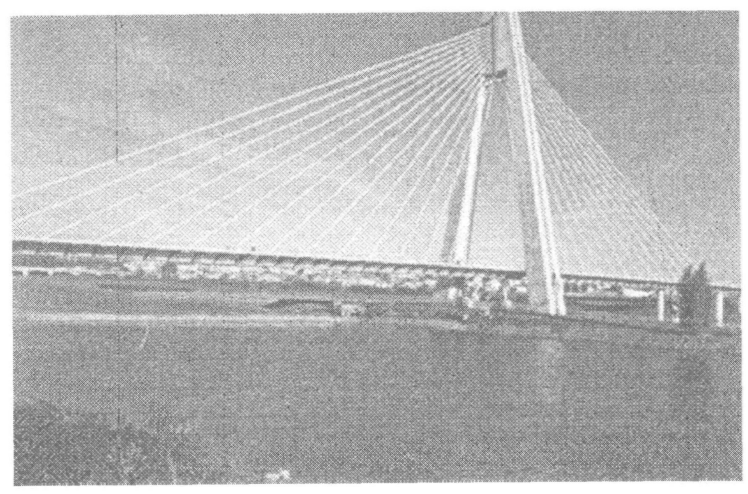

Figura 9.- Puente sobre el Guadiana a su paso por Badajoz.

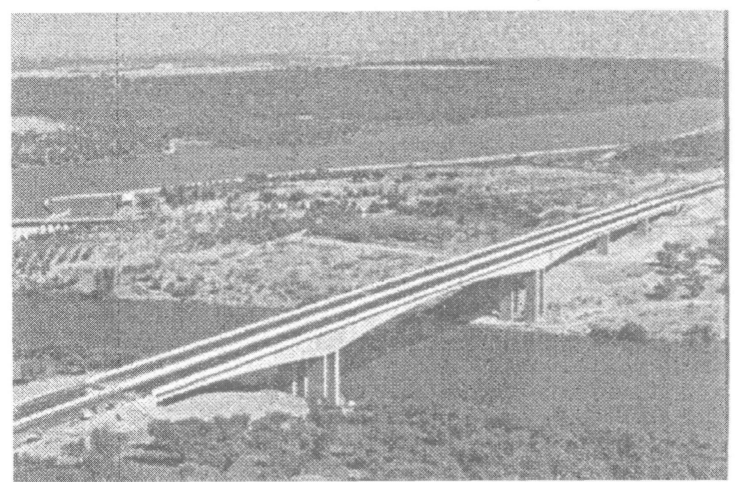

Figura 10.- Viaducto sobre el Tajo en Almaraz. ejemplo, se muestra un puente de carretera con la máxima longitud continua en España (175 metros), en la figura 10.

Finalmente, las luces muy grandes se abordan mediante puentes colgantes. Esta tipología aprovecha al máximo la eficiencia del acero para resistir tracciones y reduce al mínimo las cargas gravitatorias. Esto conduce, una vez más, al puente atirantado si, al comienzo, se invierte el arco, convirtiendo los esfuerzos de compresión en tracciones y el hormigón en acero.

En resumen, todas las tipologías estructurales se pueden aplicar y así lo han sido, en el diseño de puentes. Sin embargo, la longitud máxima es una variable clave, entre otras, para seleccionar la tipología más económica para un proyecto concreto. A pesar de ello, existe una frontera difusa entre las tipologías más adecuadas para un proyecto concreto. Por ejemplo, se pueden llegar a confundir los arcos muy rebajados y las vigas de canto longitudinalmente variable de los puentes pretensados levantados en las décadas pasadas. Para el futuro, según se ha indicado anteriormente, se prevén reducciones en las secciones, tanto longitudinal como transversalmente, y una mayor imbricación de los materiales.

\section{Análisis}

Ambos aspectos del análisis -el experimental y el numéricose han desarrollado extraordinariamente en las últimas décadas; la revolución de los computadores, el desarrollo del Método de los Elementos Finitos y de las técnicas de cálculo automatizado, así como el empleo de dispositivos electrónicos sofisticados de control de entrada y de medición de respuestas, junto con los avances en las Ciencias de los Materiales y en la teoría de la Mecánica de los Medios Continuos son algunas causas destacables de este desarro11 .

Los análisis numéricos suelen ser preferibles a los experimentales por su disponibilidad, bajo coste y rapidez de resultados. No obstante, en casos de innovaciones estructurales o de dimensiones muy grandes, es recomendable emplear ambos métodos de análisis puesto que los modelos experimentales pueden poner de manifiesto algún aspecto inesperado de comportamiento, y que, incluso, no detecte el análisis numérico, dado que éste sólo responde a las cuestiones propuestas por sí mismo. Un ensayo en un túnel de viento podría evitar algunos fallos en puentes colgantes. Por otra parte, los ensayos en modelos podrían ayudar a considerar aspectos ajenos a la estabilidad estructural, como la estética, funcionalidad o proceso constructivo. En cualquier caso, las pruebas de carga en el puente terminado han de ser obligatorias.

En un artículo sobre el estado del arte de los métodos numéricos [6] se concluyó que los modelos numéricos 
pueden simular, prácticamente, cualquier respuesta de una estructura, tanto en régimen lineal como no lineal. En cambio, se dan mayores fuentes de incertidumbre en los datos relativos a las propiedades del material (modelos de comportamiento, coeficientes elásticos y plásticos, fluencia, etc.) y a la definición de las cargas. Ello indica que el análisis de las estructuras y, por ende, el de puentes, se ha de expandir hacia ramas tales como los métodos de Identificación, teoría de la Fiabilidad, Optimización y, como consecuencia, hacia desarrollos de Sistemas Expertos de puentes.

En el diseño de puentes, el análisis ha de seguir las diferentes etapas de la propia vida del puente: construcción, servicio y carga última. Esto quiere decir que se trata de un proceso evolutivo y, por tanto, las tensiones en un instante dado dependen, en gran medida, de la historia real de las acciones aleatorias soportadas por el puente durante las etapas anteriores a la de estudio. Una alternativa para obtener una simulación aproximada de la respuesta del puente consiste en evaluarla a través de un conjunto de reglas preestablecidas -denominado formato- y dado por los diversos Códigos y Normas. El objeto de dichas reglas es, más bien, procurar un nivel de seguridad similar para todos los puentes durante las diferentes etapas de su vida útil, de acuerdo con su importancia y con otras consideraciones sociales; por el contrario, dichas reglas no pretenden simular la respuesta real de los puentes, sino obtener formatos razonables que permitan evaluar niveles similares de seguridad estructural sin necesidad de exhaustivos ensayos e investigaciones. Los coeficientes de seguridad que se incluyen en los formatos actuales proceden de una larga trayectoria y de un largo respaldo estadístico, en el sentido de que las estructuras diseñadas apropiadamente son razonablemente seguras, sin más indicación cuantitativa del nivel de seguridad alcanzado.

La tendencia futura de la simulación en estructuras se orienta hacia la unificación de los Códigos y Normas -alguna tentativa FIP-CEB ya está en curso- y hacia el establecimiento de formatos de seguridad estructural más fiables y con base científica. También se prevé el desarrollo de sistemas expertos acordes con los Códigos y Normas; esto implicará el empleo de técnicas de optimización, como parte de Sistemas basados en el Conocimiento, de pre y postprocesadores eficientes y de fácil manejo que reduzcan el camino entre el cálculo y el documento final del proyecto.

\section{Construcción}

La construcción de un puente exige una gran cantidad de trabajo costoso fuera de la fábrica. Durante un tiempo se orientaron muchos esfuerzos hacia la prefabricación. En el futuro, ésta aumentará su cuota de participación en la construcción de puentes. Dicha tendencia comenzó por el tablero, con vigas en doble $\mathrm{T}$ prefabricadas, $\mathrm{y}$ hormigonado in situ del tablero. Después, los tableros pasaron a ser multicelulares y completamente prefabricados, al igual que otras partes del puente, tales como pilas, estribos y muros de contención. Desde los puentes biapoyados iniciales, ya se construyen hoy en día en nuestro país puentes de vigas continuas, de sección variable longitudinalmente y vanos de 80 metros. Incluso el tablero de un puente atirantado se ha construido en fábrica. La referencia [7] describe el estado del arte en prefabricación en España.

En cuanto a la construcción in situ, la tendencia va hacia la mejora y automatización de los procesos constructivos. El ordenador llegará a ser una herramienta corriente para el contratista en la obra.

Ahora mismo, se pueden clasificar en tres grupos los métodos de construcción de puentes: por etapas, empujados y por voladizos. En la construcción vano a vano, hay algunas tendencias distintas, aunque suelen tener en común que la sección transversal se construye en varias etapas: normalmente se fabrica un núcleo y luego, una vez puesto en su lugar, se emplea como soporte estructural para adosarle los voladizos laterales. A veces el tablero se divide en segmentos, que pueden ser colocados (Figura 11) por procedimientos diferentes (pilas provisionales, grúas, etc.). Un ejemplo es el viaducto sobre el río Arnoya (Orense), en el que han finalizado sus dos tableros de 100 metros de longitud cada uno, rectos en planta y construidos por este procedimiento.

La técnica de empujado se ha empleado satisfactoriamente en numerosas ocasiones, hasta convertirse en un método estándar de construcción en Europa. Como es sabido, consiste en empujar grandes secciones del tablero que deslizan sobre las pilas, mediante el empleo de rodillos o elementos de teflón. El puente se fabrica o se ensambla en una zona situada en uno o en ambos extremos de la estructura $\mathrm{y}$, por tanto, se puede empujar desde uno o desde ambos extremos hacia el centro. Algunas variaciones de este método consisten en una traslación lateral del puente, o en

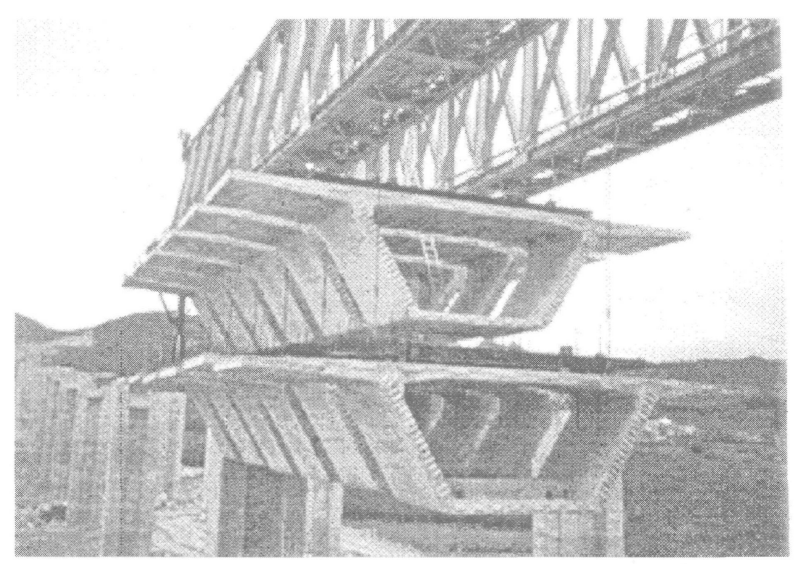

Figura 11.- Viaducto sobre el río Arnoya (Orense). 
una rotación respecto de un eje provisional. En particular, esta última permite la construcción, sin interferir con el tráfico o con el río. Un ejemplo de este método es el puente de Ben-Ahin, atirantado de una sola pila, de 168 metros de largo construido en Bélgica.

El método de los voladizos (Figura 12) se emplea a menudo cuando es preciso no interferir con el tránsito circulante bajo el puente. Es un procedimiento bastante usual, y además los récords actuales de puentes largos y muy largos han sido logrados mediante esta técnica.

\section{Función y estética del puente}

Históricamente se han utilizado algunos puentes para funciones distintas de la original. Ha servido en ocasiones como vivienda, como restaurante de carretera oúnicamente como pasarela (Figura 13). Además, el puente puede estar insertado en la ciudad, es más, puede llegar a ser el símbolo de la ciudad. El Golden Gate representa un ejemplo paradigmático. Estas consideraciones llevan a una visión del puente como obra arquitectónica y no sólo ingenieril.

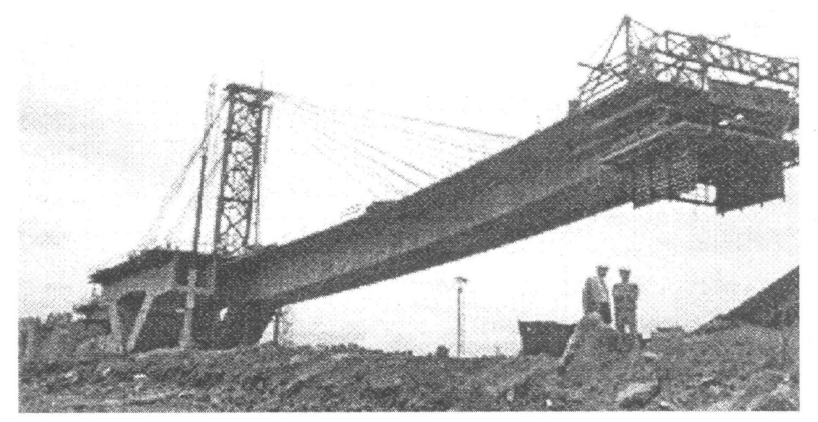

Figura 12.- Puente sobre la Ría de Pontevedra.

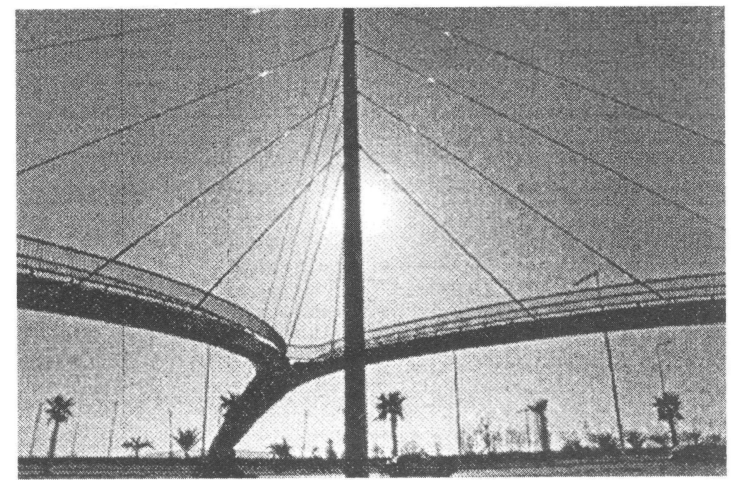

Figura 13.- Puente peatonal atirantado en Las Glorias, Barcelona.
La belleza y el placer son ingredientes necesarios del puente, sobre todo si es urbano. Pero la estética y la eficiencia técnica no son términos contradictorios, sino que a veces van juntos en un trabajo auténtico. Algunos de los puentes presentados en este documento son técnicamente impresionantes, pero no necesariamente bellos. La belleza está a menudo ligada a la armonía con el entorno y a la quietud. La pasarela de granito Inachus construida en Beppu (Japón) puede ser un ejemplo de dichas cualidades (Figura 14). Fue diseñada por M. Kawaguchi, y además de su belleza, presenta un aspecto muy interesante respecto del uso del viejo granito y del moderno pretensado. Quizá esta combinación es el símbolo de un país que aúna la tradición y el progreso igualmente.

\section{Explotación de los puentes}

Al igual que todo, los puentes tienen una vida útil y, por ello, deberían ser tratados y cuidados para que su rendimiento sea satisfactorio. Su herencia debería conservarse, al menos para aquéllos que lo merecen. Esto significa que el ingeniero no termina su trabajo con el proyecto y la construcción delpuente. El puente demanda más atención: mantenimiento, reparación y, en definitiva, explotación. En primer lugar, se debería prestar especial cuidado a situaciones ocasionales de tráfico extraordinario. La pronta detección de daños y su reparación, sin causar apenas alteraciones en el tránsito son condiciones necesarias para una buena explotación. Ya se pueden realizar pruebas y ensayos, "no invasivos", de bajo coste para detección rápida de mal comportamiento de un puente. Algunas pruebas de ese tipo están actualmente en uso; se pueden realizar sin necesidad de cortar el tránsito, cuyo ruido se filtra en el ensayo dinámico del puente (mediante modelos ARMA y análisis en el dominio del tiempo, en lugar de en el dominio de la frecuencia). En este sentido, sería deseable disponer de un expediente del puente que incluyera alguna información acerca de su comportamiento dinámico (modos y frecuencias propias de vibración) antes de que entre en funcionamiento. Dicha

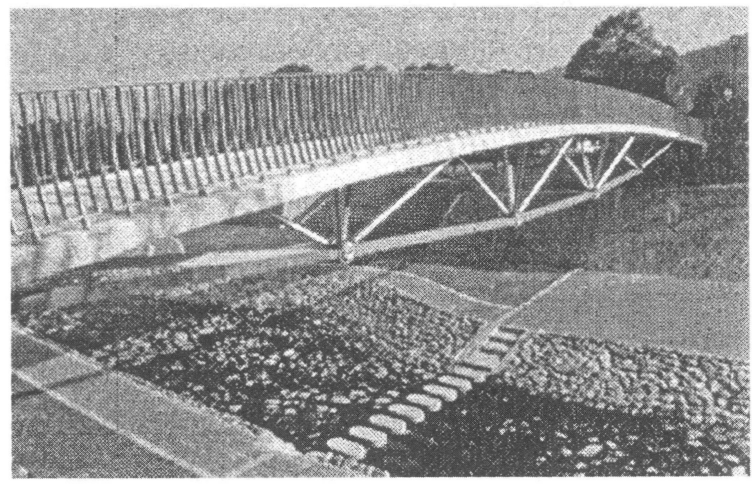

Figura 14.- Puente Inachus en Beppu (Japón). 
información se consideraría como la referencia para contrastar posteriormente el deterioro del puente durante las diferentes etapas de su vida.

\section{Observaciones finales}

Los puentes representan una estructura espacial muy simbólica que conectan con una de las necesidades primarias de la sociedad: la comunicación. Sus avances y desarrollos continuados se han producido gracias a la resolución de diferentes problemas: el reto de las grandes luces y la competición interna entre las diferentes soluciones para un mismo proyecto.

El progreso en los puentes estará estrechamente ligado al desarrollo simultáneo de los ingredientes que existen en la tecnología de los puentes, o sea, los materiales, las tipologías estructurales, el análisis, los procedimientos constructivos y la explotación. El puente del futuro, sobre todo el urbano será considerado no sólo como una obra ingenieril, sino, sobre todo, como una expresión estética del espíritu humano.

En este sentido existen asociaciones internacionales, que congregan a arquitectos, ingenieros, constructores, contratistas e investigadores. Los grupos de trabajo abordan algunos aspectos de los ingredientes anteriormente citados, pero sería precisa la creación de nuevos grupos de trabajo vinculados a aspectos de estética, reparación y mantenimiento de estructuras, técnicas de construcción y de conservación de edificaciones históricas, con vistas a identificar y encontrar respuesta, de una manera amplia, a la problemática que surge en la tecnología de los puentes. La coordinación entre todos los grupos de trabajo sería de todo punto necesaria.

Existe una gran cantidad de literatura relacionada con los diferentes aspectos de la tecnología de los puentes. Además de las ya citadas, se recomiendan las referencias [8] a [15] para mayor profundización.

\section{Agradecimientos}

El autor desea expresar su reconocimiento público de agradecimiento por la ayuda en la documentación recibida en la preparación de este artículo a las siguientes personas e instituciones: A.J. Reis (IST, Lisboa), S. Pérez Fadón (FERROVIAL), J. Manterola (Carlos Fernández Casado OT), M. Juliá (CUBIERTAS), A. Scordelis (Univ. of California at Berkeley), P.L. Gould (Washington Univ. in St. Louis, USA), J.I. González-Esteban (FOMENTO), M. Kagaguchi (Hosei Univ. Tokyo), J.J. Arenas (APIA XXI), M. Burón (PACADAR) y L. Albajar (ALVISA).

Este artículo representa un resumen de la Conferencia impartida por el autor dentro del ciclo "Current and emergent technologies of shell and spatial structures", celebrado en el
CEDEX del 28 al 30 de abril de 1998, bajo los auspicios de la IASS (International Association for Shell and Spatial Structures). Asimismo, el autor desea expresar su agradecimiento por la preparación y traducción de este artículo al Dr. Ing. de Caminos Juan Carlos Mosquera Feijóo (U.P.M.)

\section{BIBLIOGRAFÍA}

[1] WITTFOHT, H.: "Triumph der Spannweiten" Beton Verlag, GmBH, Düsseldorf (1971) pp.10.

[2] SCORDELIS, A.C.: "Cable-suspended bridges- Past, Present and Future" ASCE Structures Congress, SF Lecture, (May 4, 1989).

[3] APARICIO, A.: "Los puentes del futuro". OP Núm. 20 (1991) pp 6-21.

[4] REIS, A.J.: "Recent developments in composite bridges" in Steel Structures. Eurosteel'95. A.N. Kounadis (Ed). Balkera/Rotterdam. (1995). pp 383-389.

[5] TORROJA, E.: "Razón y Ser de los tipos estructurales". Instituto Técnico de la Construcción. Madrid. $2^{\mathrm{a}}$ Ed. (1960). pp-101.

[6] SAMARTIN, A.: "Numerical analysis of shells and space structures". Bulletin of the International Association for Shell and Spatial Structures. Vol 33, N 13 (n. 107) December(1991). pp181-188.

[7] LLEYDA, J.L.: "La prefabricación en obras civiles. Estado del arte en España". Hormigón y Acero. Núm. 198. Madrid (1995) pp 127-144.

[8] LEONHARDT, F.: "Brücken. Ästhetik und Gestaltung. Bridges. Aesthetics and Design". The Architectural Press: London(1982)

[9] WALther, R. and al.: "Ponts Haubanés". Presses Polytechniques Romandes (1985).

[10] GIMSING, N.J.: "Cable supported Bridges. Concept and Design". John Wiley and Sons (1983).

[11] PODOLNY, W. and J.B. SCALZI: "Construction and Design of Cable-stayed Bridges". 2nd. edition. John Wiley and Sons (1986).

[12] ZIENCKIEWICZ, O.C. and Y.K.CHEUNG: "The finite element method for analysis of elastic isotropic and orthotropic slabs". Proc. Inst. Civ. Eng. 28 (1964) pp 471-88.

[13] SAMARTÍN, A. Q.: "Cálculo de estructuras de puentes de hormigón”. Ed. Rueda. Madrid (1983).

[14] ZIENCKIEWICZ, O.C. and R.L. TAYLOR: "The Finite Element Method". 4th edition McGraw-Hill Book Co.Proc London (1989).

[15] SCORDELIS, A.C. et al.: Reports no. UCB/SESM 76-6, 78-2, 79-3, 84-12, 86-7 and 86-13. Department of Civil Engineering. University of California. Berkeley, California. 